\title{
„Hamburg in Bewegung“. Räumliche Mobilität und die Arbeitsmarktintegration unter sozialversicherungspflichtig Beschäftigten in Hamburg
}

\author{
Volker Kotte ${ }^{1}$
}

Eingegangen: 21. Mai 2015 / Angenommen: 17. Februar 2016 / Online publiziert: 10. März 2016

(C) Springer-Verlag Berlin Heidelberg 2016

\begin{abstract}
Zusammenfassung Berufsbedingtes Pendeln ist ein wachsendes Phänomen. Eine immer größere Zahl an Beschäftigten legt dabei immer weitere Wege zwischen Wohn- und Arbeitsort zurück. Die Untersuchung beschäftigt sich mit dem Zusammenhang zwischen Pendeln und der Integration in den Arbeitsmarkt. Auch die Umzugsbereitschaft der Beschäftigten wird vor dem Hintergrund personenbezogener Merkmale untersucht. Dazu werden die Daten von rund 730.000 sozialversicherungspflichtig Beschäftigten, die am 30.06.2000 einen Arbeits- oder Wohnort in Hamburg hatten, über einen Zeitraum von 13 Kalenderjahren betrachtet. Die Ergebnisse sind differenziert: Einpendler sind hinsichtlich der Arbeitsmarktintegration recht erfolgreich, aber Pendler nicht generell erfolgreicher als Nichtpendler, wie am Beispiel der Auspendler deutlich wird. Die Arbeitsmarktintegration hat einen Bezug zu räumlicher Mobilität und deren zeitlicher Dynamik, der Zusammenhang ist aber nicht in jedem Fall eindeutig. Im Hinblick auf die Umzugsbereitschaft der sozialversicherungspflichtig Beschäftigten konnten die Ergebnisse der Mobilitätsforschung im Wesentlichen bestätigt werden.
\end{abstract}

Schlüsselwörter Pendler · Räumliche Mobilität · Arbeitsmarktintegration · Wohnsitzentscheidung · Hamburg

Dr.Volker Kotte

volker.kotte@iab.de

Institut für Arbeitsmarkt- und Berufsforschung der

Bundesagentur für Arbeit,

Projensdorfer Str. 82, 24106 Kiel, Deutschland

\section{"Hamburg in Motion". Commuting and labour market integration among employees in Hamburg}

\begin{abstract}
The number of persons commuting to and from work is steadily increasing. In this paper, commuting patterns in the city of Hamburg, Germany, are examined over a period of 13 years. Drawing on unique career data of 730,000 persons who were employed or lived in Hamburg in the year 2000, (1), in-, out-, and non-commuters are compared with regard to labor market outcomes such as employment and unemployment. (2), the rationales for decisions of commuters to relocate their place of residence nearby the workplace are analyzed. It becomes apparent that those who commute to Hamburg are more often employed than those who do not commute. Non-commuters, however, are more successful on the labor market than those who commute from Hamburg to workplaces outside the city so that the benefits of commuting do not outweigh its costs for all types of mobility between residence and workplace. Finally, moving to the workplace region is depending on several personal characteristics. The findings mainly verify the results of existing mobility studies.
\end{abstract}

Keywords Commuting $\cdot$ Mobility $\cdot$ Labor market integration $\cdot$ Residential location decision $\cdot$ Hamburg

\section{Forschungsstand und Fragestellung}

Immer mehr Berufstätige pendeln täglich zwischen Wohnund Arbeitsort. ${ }^{1}$ Nicht nur die Zahl der Pendler, auch die dabei zurückgelegte Entfernung nimmt zu (exemplarisch für die

\footnotetext{
${ }^{1}$ Die zirkuläre räumliche Mobilität, das heißt das Auseinanderfallen von Arbeits- und Wohnort, wird im Text als Pendeln bezeichnet. Im Text wird überwiegend die männliche Sprachform verwendet.
} 
Literatur: Kalter 1994; Einig/Pütz 2007: 73; Haas/Hamann 2008; Granato/Haas/Hamann et al. 2009; Ruppenthal/Lück 2009; Guth/Siedentop/Holz-Rau 2012; Pfaff 2012; Pfaff 2013; Pfaff 2014; in Bezug auf Hamburg auch Holtermann/Otto/ Schulze 2013). In der sozialwissenschaftlichen Literatur wird das Phänomen des berufsbedingten Pendelns mit gesellschaftlichen Modernisierungsprozessen in Verbindung gebracht, insbesondere in der Arbeitswelt aber auch mit dem allgemeinen sozialen Wandel (exemplarisch für die Modernisierungsdebatte van der Loo/van Reijen 1992 oder Beck/Bonß 2001).

Setzt man sich mit beruflichem Pendeln auseinander, kommt die Frage auf, warum Arbeitnehmer bereit sind, zwischen Wohn- und Arbeitsort größere Distanzen zurückzulegen. Pendeln ist mit „Kosten“ verbunden. Einige davon liegen ökonomisch klar auf der Hand, etwa Ausgaben für Verkehrsmittel oder notwendige Dienstleistungen, andere sind monetär schwer zu quantifizieren, aber nicht weniger bedeutend - etwa gesundheitliche oder soziale Belastungen im Zusammenhang mit dem Pendeln. In den Sozialwissenschaften existieren verschiedene Ansätze, um die Bereitschaft zu räumlicher Mobilität zu erklären. Eine besondere Bedeutung kommt mikrostrukturellen Ansätzen zu, die zur Theorietradition der rationalen Entscheidung gehören (,rational choice").

Pendeln ist im handlungstheoretischen Verständnis eine individuelle Entscheidung, in der Kosten und Nutzen der Varianten abgeglichen werden und diejenige mit der höchsten Werterwartung ausgewählt wird. Diese Annahme hat hohe Erklärungskraft, etwa wenn Mobilität mit Einkommensverbesserungen, beruflichem Aufstieg oder der Abwendung von Arbeitslosigkeit in Verbindung gebracht wird. Aber auch andere, eher qualitative Aspekte lassen sich integrieren, z. B. der Wunsch nach einer beruflichen Neuorientierung oder familienbedingte Umzüge. Verhandlungstheoretische Positionen erweitern das Spektrum der Entscheidungsfindung auf mehrere Beteiligte. Sie gehen von dem Paradigma aus, dass Entscheidungen nicht allein, sondern nur in Abstimmung mit Anderen getroffen werden können. Auch diese Erweiterung besitzt hohe Erklärungskraft, denn tatsächlich dürfte die Entscheidung zum Pendeln sehr oft in Abstimmung mit Partnern, Kindern oder anderen Haushaltsangehörigen getroffen werden. Ein weiterer Erklärungsansatz bindet räumliche Mobilität in den biographischen Lebenszyklus des Einzelnen ein. Dahinter steht die Annahme, dass berufliche Ereignisse durch die Stellung im Lebenszyklus wesentlich mitbestimmt werden. Der Eintritt in das Berufsleben, Familienphasen mit Kindern oder der Übergang in den Ruhestand sind Beispiele für Statuspassagen, die Auswirkungen auf räumliche Mobilität haben können (für eine Übersicht hierzu vergleiche Pfaff 2013: 18 ff. oder Frick 1996: 27 ff.).

Pendeln gilt entscheidungstheoretisch als KostenNutzen-Abwägung. Die Entscheidung zu pendeln müsste demnach mit einer höheren Nutzenerwartung verbunden sein. Abraham und Nisic (2007) gingen dem Zusammenhang zwischen Pendeln und der Arbeitsmarktplatzierung in Deutschland und der Schweiz nach (Abraham/Nisic 2007: 75). Für beide Länder ist ein positiver Effekt zwischen regionaler Mobilität und der Höhe des Einkommens nachzuweisen.

In diesem Beitrag steht der Zusammenhang zwischen räumlicher Mobilität und der Arbeitsmarktintegration im Mittelpunkt. Nach einer Beschreibung des Datensatzes und der Untersuchungsregion in den folgenden Kapiteln 2 und 3 wird im 4. Kapitel die Integration in den Arbeitsmarkt in Verbindung mit der Pendlermobilität diskutiert: Betrachtet wird Pendeln in Bezug zu Zeiten in sozialversicherungspflichtiger Beschäftigung und Zeiten in Arbeitslosigkeit. Nutzenmaximierend ist anzunehmen, dass Pendler dabei besser abschneiden als Nicht-Pendler. In Bezug auf berufsbedingtes Pendeln wird häufig auch die Frage nach dem Wechsel des Wohnortes gestellt. Der Zusammenhang zwischen der Umzugsbereitschaft und der beruflichen Situation wurde schon in vielen anderen Studien untersucht. Windzio (2004: 39 ff.) konnte in seiner Analyse von Fernwanderungen - neben weiteren Faktoren - einen positiven Zusammenhang zwischen Einkommen, Bildungsgrad und der Bereitschaft zu räumlicher Mobilität nachweisen. Auch demographische Faktoren wie Alter oder Familienstand spielen eine wichtige Rolle, wie etwa Kley (2009: $167 \mathrm{ff}$.) nachweisen konnte. Frick (1996: 215 ff.) ist in einer breit angelegten Untersuchung in Bezug auf die genannten Merkmale zu ähnlichen Ergebnissen gekommen. Kalter (1997) hat Wanderungen in ein umfassendes dreistufiges Prozessmodell aus Entstehung, Planung und Realisierung eingebettet. In der vorliegenden Analyse wird deshalb in Kapitel 5 auch die Umzugsbereitschaft sozialversicherungspflichtig Beschäftigter betrachtet. Dabei geht es um die Suche nach personenbezogenen Merkmalen, die in diesem Kontext eine Rolle spielen können. Abschließend werden die Ergebnisse im Kapitel 6 zusammengefasst und diskutiert.

\section{Datengrundlage und Untersuchungsregion}

Datengrundlage sind die integrierten Erwerbsbiographien (IEB) des Instituts für Arbeitsmarkt- und Berufsforschung. Die IEB enthalten tagesgenaue und personenbezogene Daten zur Beschäftigung, dem Bezug von Entgeltersatzleistungen, Zeiten der Arbeitslosigkeit oder Arbeitssuche sowie Maßnahmen der Arbeitsförderung. Vereinfacht gesagt werden Meldungen der Betriebe und Daten aus der Arbeitsverwaltung für jede Person als Kontenverlauf verfügbar gemacht. Insgesamt werden etwa zwei Drittel der Erwerbstätigkeit in Deutschland durch die IEB erfasst (Bundesagentur für Arbeit 2014). Die aktuelle Version der IEB (V11.00.00) enthält Informationen zu rund 82 Mio. 
Personen, die ältesten Datensätze reichen bis in das Jahr 1975 zurück (für eine Beschreibung vergleiche Kotte 2011).

Im Untersuchungspanel enthalten sind 728.450 sozialversicherungspflichtig Beschäftigte, die am 30.06.2000 entweder einen Arbeits- oder Wohnort in Hamburg hatten. Aufgenommen wurden sozialversicherungspflichtig Beschäftigte (statistischer Status „101“), Personen in Altersteilzeit (Status „103“) und Beschäftigte in der Seefahrt (Status „140“) unabhängig von ihrer Arbeitszeit. Ausgeschlossen wurden Auszubildende (duale Ausbildung), Personen in Qualifizierungsmaßnahmen (insbesondere Werkstudenten und Praktikanten), geringfügig Beschäftigte (Minijobber) und mithelfende Familienangehörige. Insbesondere unter Auszubildenden und Minijobbern existieren Mobilitätsmuster, etwa die Häufung von Ortswechseln zu Beginn oder am Ende der Ausbildung, die einen Ausschluss aus dem Panel rechtfertigen.

Ausgehend von diesen 728.450 Beschäftigten wurden alle Kontoinformationen dieser Individuen in den Kalenderjahren 2000 bis 2012 bereitgestellt. Im Idealfall liegt ein lückenloser Berufsverlauf über 13 Kalenderjahre vor, im ungünstigsten Fall nur ein einziger Beschäftigungstag (das wäre dann der Ausgangsstichtag, der 30. Juni 2000). ${ }^{2}$ Die Auswahl des 30. Juni folgt vorhandenen Auswertungen, ist aber auch in saisonaler oder meteorologischer Hinsicht für Beschäftigungsanalysen vergleichsweise günstig. Alle angegebenen Zeiträume beruhen auf Kalendertagen, auch wenn im Text von Beschäftigungszeiten oder Tagen in Arbeitslosigkeit die Rede ist. Der Gesamtzeitraum vom 01.01.2000 bis zum 31.12.2012 umfasst 4.749 Kalendertage (einschließlich der Schalttage).

Einschränkend muss gesagt werden, dass die Operationalisierung des Pendelns durch die Überschreitung der Stadt- bzw. Landesgrenze mit konzeptionellen Problemen verbunden ist. Kleine Gemeinden mit wenigen Einwohnern und einer geringen Dichte an Arbeitsplätzen stehen auf derselben Stufe mit Metropolen wie Hamburg. ${ }^{3}$ Auch innerhalb der Hamburger Stadtgrenzen existiert räumliche Mobilität, die quantitativ und qualitativ den Vergleich mit der überregionalen nicht zu scheuen braucht. ${ }^{4}$ Das gewählte Untersuchungsdesign folgt gleichwohl

\footnotetext{
${ }^{2}$ Beim Merkmal Arbeitslosigkeit kann eine Episode auch vor dem 01. Januar 2000 beginnen. Die kalendarische Dauer kann von der rechtlichen Arbeitslosigkeit abweichen, was z. B. durch Krankheit oder fehlende Verfügbarkeit verursacht sein kann. Beginnt eine Arbeitslosigkeitsepisode vor dem 01.01.2000, ist die Aufteilung nach schädlichen und unschädlichen Unterbrechungen für die Jahre 1999 und 2000 nicht rekonstruierbar, diese Fälle werden daher mit ihrer gesamten Episode in die Untersuchung einbezogen.

${ }^{3}$ Für die Dichte an Arbeitsplätzen im schleswig-holsteinischen Umland vergleiche Schulz/Bröcker 2007.

${ }^{4}$ Für die räumliche Mobilität im nördlichen Umfeld Hamburgs vgl. Herrmann/Schulz 2005; Schulz/Bröcker 2007 und Herrmann 2009; siehe auch Holtermann/Otto/Schulze 2013.
}

existierenden Abgrenzungen und Statistiken und ist - nicht zuletzt - auch arbeitsökonomischen Motiven geschuldet.

Hamburg hat eine überregionale Bedeutung für den norddeutschen Arbeitsmarkt. Die Stadt ist Handels-, Verkehrsund Logistikzentrum für Norddeutschland, Sitz großer Unternehmen und bedeutender Medien- und Bildungsstandort. Der Hamburger Arbeitsmarkt zeichnet sich durch eine hohe Dynamik aus: Zwischen 2000 und 2015 ist die Zahl der sozialversicherungspflichtig Beschäftigten um rund 108.000 auf heute etwa 912.000 Personen gewachsen (Beschäftigte am Arbeitsort, Stichtag jeweils der 30.06.). Dabei bestehen zwischen der Stadt und ihrem Umland enge Verflechtungen. Aktuell wohnt etwa jeder vierte Beschäftigte außerhalb (Bundesagentur für Arbeit 2016; zum Hamburger Arbeitsmarkt und zur räumlichen Verflechtung in der Metropolregion vgl. auch Kotte 2008; Niebuhr/Kotte 2009 oder Buch/ Hamann/Niebuhr 2010). Insgesamt hat berufsbedingtes Pendeln in den letzten Jahren an Bedeutung gewonnen. Einig und Pütz (2007: 73 ff.) konnten in einer mehrjährigen Analyse - auch für den Hamburger Raum - ein Wachstum der Pendlerzahl und der dabei zurückgelegten Entfernungen nachweisen.

\section{Das Beschäftigtenpanel: Zahlen und Daten}

ImPanel sind 728.450 Beschäftigte erfasst, die am 30.06.2000 entweder ihren Arbeits- und/oder ihren Wohnort in Hamburg hatten. Diese werden in drei Gruppen geteilt: Nichtpendler, Einpendler und Auspendler. Nichtpendler sind Beschäftigte, die in Hamburg wohnen und arbeiten; diese stellten am 30.06.2000 mit 420.131 Personen die größte Gruppe. Einpendler arbeiten in Hamburg, aber wohnen außerhalb der Stadt; dazu zählten am Stichtag 239.410 Personen. Auspendler arbeiten außerhalb Hamburgs, wohnen aber in der Stadt; sie bilden mit 69.909 Personen die kleinste Gruppe in der Untersuchung (vgl. auch Tabelle 5 im Anhang). ${ }^{5}$ Die Einteilung nach Gruppen richtet sich zunächst ausschließlich nach der Wohn- und Arbeitsortkombination am 30. Juni 2000. Spätere Änderungen führen nicht zu einem Wechsel der Pendlerkategorie. Die Frage der zwischenzeitlichen Verlagerung von Wohn- oder Arbeitsorten wird im fünften Kapitel aufgegriffen.

Insgesamt sind im Panel mit einem Anteil von 58,7\% mehr Männer als Frauen enthalten, unter den Pendlern

\footnotetext{
${ }^{5}$ Aufgrund der Struktur des Datensatzes sind Abweichungen zu den amtlichen Pendlerstatistiken möglich. Die Statistik der Bundesagentur für Arbeit weist am 30.06.2000 folgende Stichtagsdaten aus (ebenfalls sozialversicherungspflichtig Beschäftigte ohne Auszubildende): 264.627 Einpendler, 74.573 Auspendler und 539.968 Nichtpendler. Die amtlichen Zahlen basieren auf aggregierten Stichtagsdaten, diese Untersuchung auf personenbezogenen Längsschnittdaten.
} 
ist dieser Anteil mit etwa zwei Dritteln noch höher (vgl. Tabelle 5). Aus Untersuchungen ist bekannt, dass Männer weitere Pendelwege zurücklegen als Frauen (vgl. u. a. Abraham/Schönholzer 2009; Ruppenthal/Lück 2009; Feldhaus/Schlegel 2013). Dieses Phänomen steht häufig mit der Lebenslage in Verbindung. Frauen sind in höherem Maße durch Betreuungspflichten räumlich gebunden (Kinder oder pflegebedürftige Angehörige) als Männer. Aber auch die geschlechtsspezifische Segmentation des Arbeitsmarktes wirkt in diese Richtung. Frauen sind oftmals in Berufen oder Branchen tätig, die ,flächendeckend“ existieren (z. B. Handel, personenbezogene Dienstleistungen, Gesundheitswesen oder pädagogische Berufe). In Branchen oder Berufen mit hoher räumlicher Konzentration stellen überwiegend Männer die Mehrheit der Beschäftigten (etwa in der Industrie, in technischen Berufen oder unternehmensnahen Dienstleistungen).

Das Durchschnittsalter im Panel am 30.06.2000 betrug rund 39 Jahre, Männer sind dabei etwas älter als Frauen. Von der Qualifikationsstruktur her verfügen mehr als zwei Drittel über eine abgeschlossene Berufsausbildung (einschließlich Aufstiegsqualifizierungen wie Meister, Techniker, Fachwirte etc.; für die Werte vgl. Tabelle 6), ein gutes Fünftel verfügt über ein abgeschlossenes Studium $(21,3 \%)$ und lediglich 8,4\% über keinen formalen Berufsabschluss. Frauen sind dabei im unteren und mittleren Qualifikationssegment stärker vertreten als Männer, die Pendler besser qualifiziert als die Nichtpendler. ${ }^{6}$

\section{Die Arbeitsmarktintegration: Beschäftigung und Arbeitslosigkeit von Pendlern}

\subsection{Sozialversicherungspflichtige Beschäftigung}

Wie im ersten Kapitel erläutert, ist Pendeln entscheidungstheoretisch eine Kosten-Nutzen-Abwägung. Pendeln sollte subjektiv einen höheren Nutzen erwarten lassen als die Entscheidung, nicht zu pendeln. Zwar kann die individuelle Entscheidung mit den vorliegenden Daten nicht nachvollzogen werden, aber die Erwartung einer hohen Beschäftigungskonstanz und eine möglichst geringe Betroffenheit durch Arbeitslosigkeit können als wichtige Aspekte einer solchen Nutzenabwägung betrachtet werden. Räumliche Mobilität sollte in dieser Ableitung in der Gruppe mit höheren Beschäftigungszeiten und entweder keiner oder einer geringeren Arbeitslosigkeit verbunden sein.

\footnotetext{
${ }^{6}$ Das schlechtere Qualifikationsniveau der Frauen ist auch durch das Untersuchungsdesign bedingt. Ausgewertet wurden Daten von Personen, die am 30.06.2000 in einer sozialversicherungspflichtigen Beschäftigung standen. Das Qualifikationsniveau der altersgleichen Wohnbevölkerung dürfte hiervon abweichen.
}

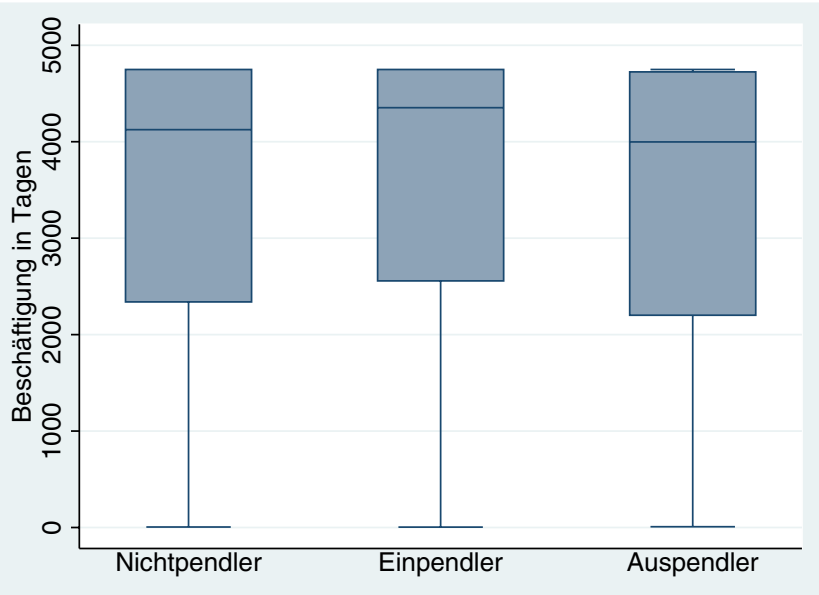

Abb. 1 Tage in sozialversicherungspflichtiger Beschäftigung (Zeitraum 01.01.2000 bis 31.12.2012). (Quelle: IEB (V11.00.00) und eigene Berechnungen)

Zunächst zur sozialversicherungspflichtigen Beschäftigung: Die drei Gruppen sind in Abbildung 1 als Kastengrafik (Boxplot) und als Survivor-Funktion (Verlaufsdatenanalyse) in den Abbildungen 2 und 3 dargestellt, die Werte finden sich in Tabelle 1.7 Im Durchschnitt befand sich jede Person an 3.500 Tagen in sozialversicherungspflichtiger Beschäftigung, das sind rechnerisch rund neuneinhalb von dreizehn Jahren (der Zeitraum vom 01.01.2000 bis 31.12.2012 entspricht 4.749 Tagen). ${ }^{8}$ Die Abweichungen zwischen den Gruppen fallen in Anbetracht des Zeitraumes klein aus, die Spanne zwischen Auspendlern und Einpendlern beträgt 192 Kalendertage. Einpendler erreichen die höchsten Beschäftigungswerte (3.586 Tage), gefolgt von den Nichtpendlern mit 3.469 Tagen und den Auspendlern mit 3.394 Tagen. Auf den ersten Blick sind das hohe Beschäftigungswerte. Dabei ist zu bedenken, dass nicht die gesamte erwerbsfähige Bevölkerung, sondern nur diejenigen, die am 30.06.2000 (bereits) in einer sozialversicherungspflichtigen Beschäftigung standen, die Ausgangsgruppe bilden. Andere Erwerbsfähige sind in der Analyse nicht enthalten. Das Untersuchungspanel ist durch den Zuschnitt auf (bereits) Beschäftigte positiv „,verzerrt““

Ein genauer Blick auf die Verteilung der Beschäftigungsdauer zeigt weitere Details. Etwa jeder Vierte im Panel ist durchgehend beschäftigt (vgl. Tabelle 1 und Abbildung 2). Im

\footnotetext{
${ }^{7}$ Ausführliche Werte zu Beschäftigung, Arbeitslosigkeit und den Wohnorten finden sich im Anhang in Tabelle 7.

${ }^{8}$ Dass bei vielen Individuen nicht der gesamte Zeitraum von 4.749 Tagen erfasst ist, liegt an den Datenquellen. Betrachtet wurden Zeiten als sozialversicherungspflichtig Beschäftigter oder Zeiten der registrierten Arbeitslosigkeit. Tätigkeiten als Selbstständiger, Freiberufler, Beamter, Richter, Soldat fehlen ebenso wie Ausbildungsoder Studienzeiten, Auslandsaufenthalte oder der Rückzug vom Arbeitsmarkt (in die sogenannte „Stille Reserve“ - vgl. zu ihrer Definition Fuchs/Walwei/Weber 2005).
} 
Abb. 2 Überlebensfunktion (Kaplan-Meier) der Tage in sozialversicherungspflichtiger Beschäftigung zwischen dem 01.01.2000 und dem 31.12.2012 (Summe aller Beschäftigungsverhältnisse)*. (Quelle: IEB (V11.00.00) und eigene Berechnungen. *Summiert werden alle Zeiten in sozialversicherungspflichtiger Beschäftigung (auch aus verschiedenen Beschäftigungsverhältnissen, unabhängig vom Pendlerstatus))

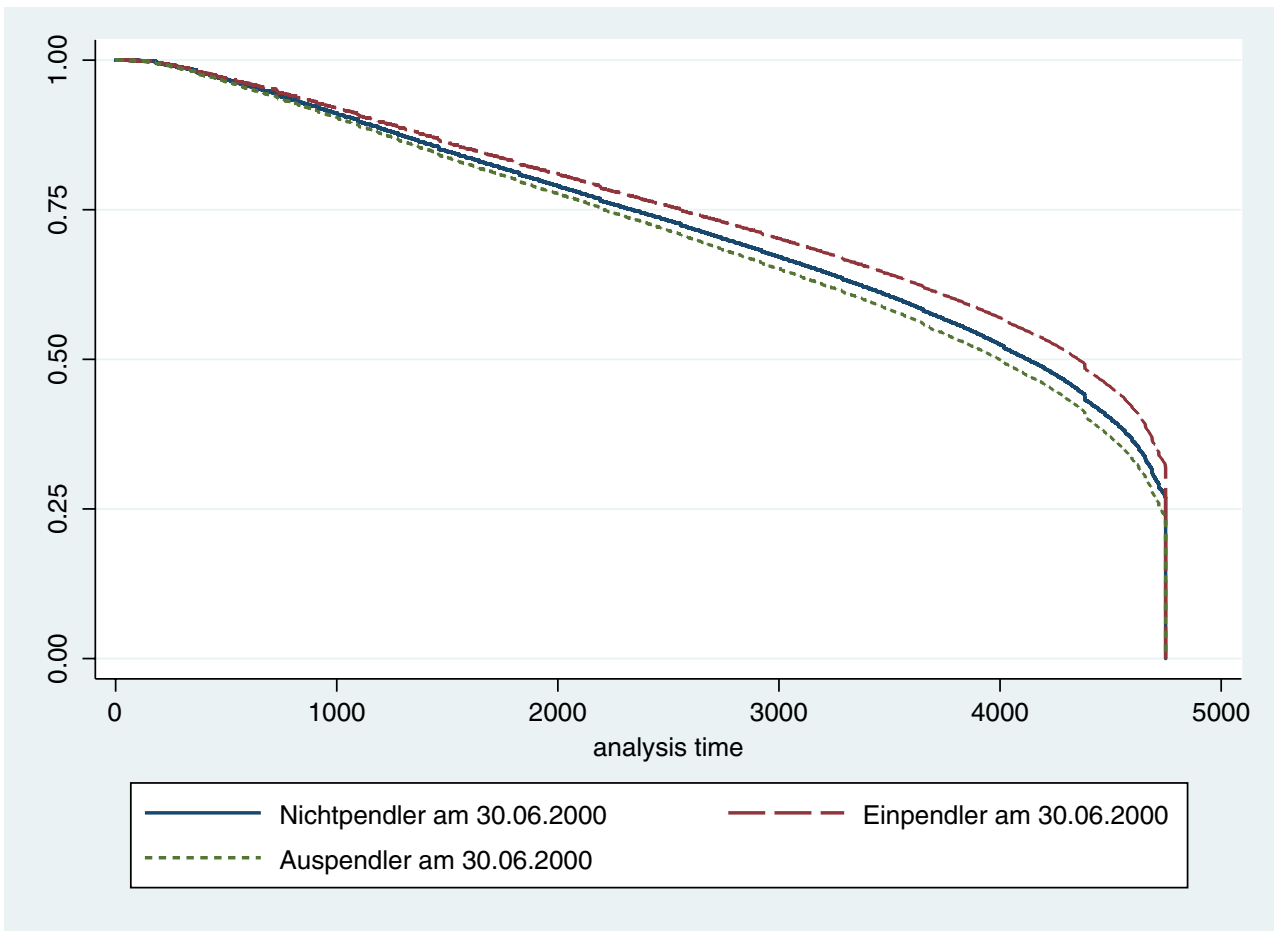

Abb. 3 Überlebens-

funktion (Kaplan-Meier) der Beschäftigungszeiten zwischen dem 01.01.2000 und dem 31.12.2012 in der Pendlerkategorie des 30.06.2000 in Tagen*. (Quelle: IEB (V11.00.00) und eigene Berechnungen. *Summiert werden Zeiten in sozialversicherungspflichtiger Beschäftigung in der Pendlerkategorie des 30.06.2000 (auch aus verschiedenen Beschäftigungsverhältnissen, aber nur im ursprünglichen Pendlerstatus))

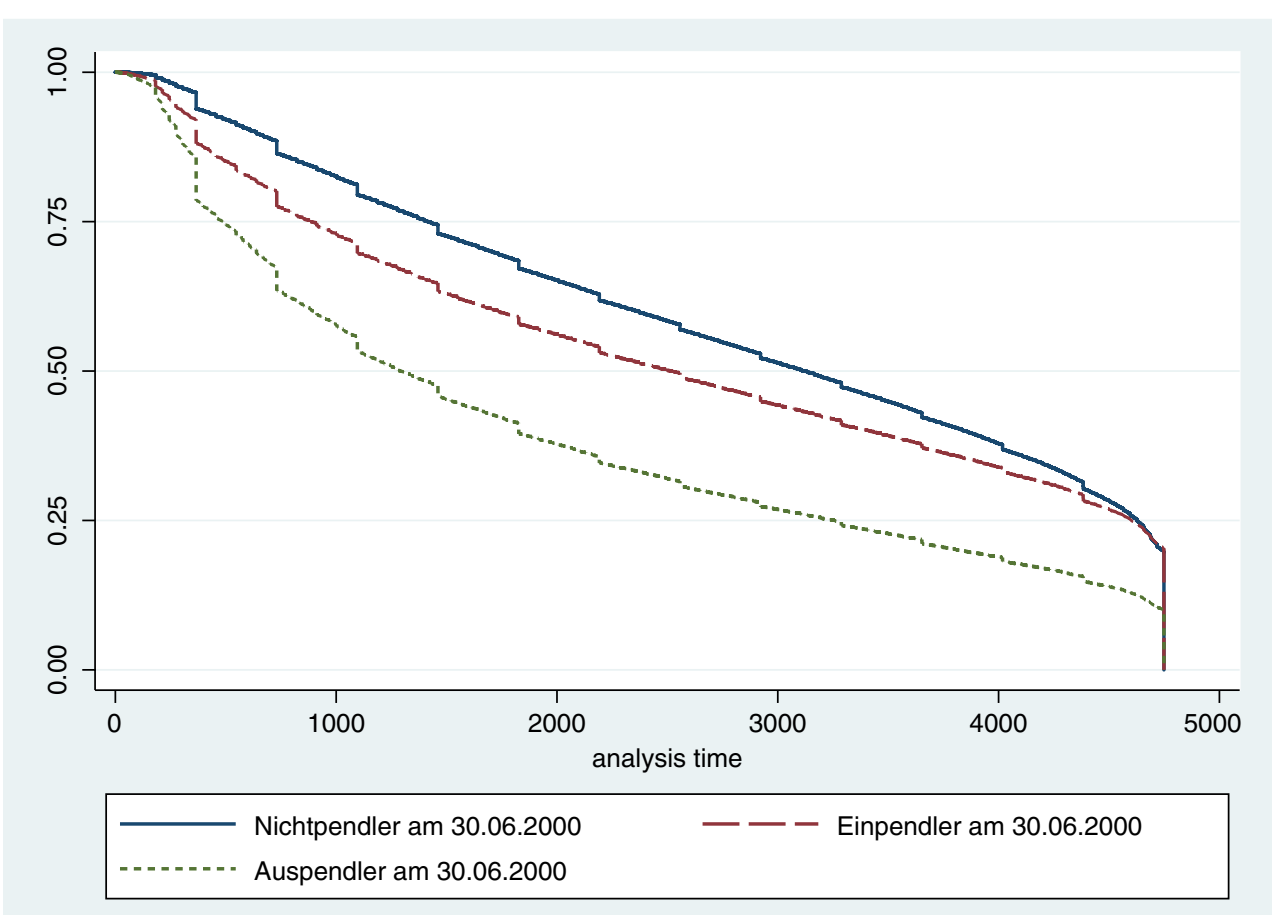

Mittel erreicht jeder Zweite rund 11,5 Beschäftigungsjahre, wobei an dieser Stelle schon Unterschiede zwischen den Gruppen sichtbar werden. Während jeder zweite Einpendler auf mindestens 4.353 Beschäftigungstage kommt, ist es in der gleich großen Gruppe der Auspendler fast ein Jahr weniger (3.998 Tage). Jenseits des Medians nehmen die Beschäftigungszeiten stärker ab. Ein Viertel erreicht noch
2.392 Beschäftigungstage, also etwa 6,5 Jahre, die untersten zehn Prozent noch rund drei Jahre und fünf Prozent sogar weniger als zwei Jahre. Aus der Abbildung 2 werden die Unterschiede im Zeitverlauf deutlich.

Zwischen der Beschäftigungsdauer und Veränderungen des Wohn- und Arbeitsortes besteht ein Zusammenhang, wie ein Vergleich der Abbildung 2 mit der Abbildung 3 zeigt. In 
Tab. 1 Beschäftigungszeiten nach Pendlergruppe. (Quelle: IEB (V11.00.00) und eigene Berechnungen)

\begin{tabular}{lcllllllll}
\hline & Anzahl & Mittelwert & $\begin{array}{l}\text { Standardab- } \\
\text { weichung }\end{array}$ & $\begin{array}{l}5 \% \%^{-} \\
\text {Perzentil }\end{array}$ & $\begin{array}{l}10 \%- \\
\text { Perzentil }\end{array}$ & $\begin{array}{l}25 \% \%^{-} \\
\text {Perzentil }\end{array}$ & $\begin{array}{l}50 \% \text { - }^{-} \\
\text {Perzentil Median }\end{array}$ & $\begin{array}{l}75 \%- \\
\text { Perzentil }\end{array}$ & $\begin{array}{l}90 \%- \\
\text { Perzentil }\end{array}$ \\
\hline Panel, insgesamt & 728.450 & 3.500 & $1.432,3$ & 677 & 1.096 & 2.392 & 4.199 & 4.749 & 4.749 \\
Nichtpendler & 420.131 & 3.469 & $1.435,9$ & 670 & 1.096 & 2.339 & 4.125 & 4.749 & 4.749 \\
Einpendler & 239.410 & 3.586 & $1.416,0$ & 731 & 1.179 & 2.557 & 4.353 & 4.749 & 4.749 \\
Auspendler & 68.909 & 3.394 & $1.450,7$ & 613 & 1.032 & 2.201 & 3.998 & 4.724 & 4.749 \\
\hline
\end{tabular}

Tab. 2 Zeiten in Arbeitslosigkeit nach Pendlergruppe. (Quelle: IEB (V11.00.00) und eigene Berechnungen)

\begin{tabular}{lllllllll}
\hline & Anzahl & Mittelwert & $\begin{array}{l}\text { Standardab- } \\
\text { weichung }\end{array}$ & $\begin{array}{l}50 \%- \\
\text { Perzentil Median }\end{array}$ & $\begin{array}{l}75 \%- \\
\text { Perzentil }\end{array}$ & $\begin{array}{l}90 \%- \\
\text { Perzentil }\end{array}$ & $\begin{array}{l}95 \%- \\
\text { Perzentil }\end{array}$ & $\begin{array}{l}99 \%- \\
\text { Perzentil }\end{array}$ \\
\hline Panel, insgesamt & 728.450 & 208,0 & 463,0 & 0 & 193 & 697 & 1.170 & 2.315 \\
Nichtpendler & 420.131 & 220,3 & 481,7 & 0 & 212 & 731 & 1.245 \\
Einpendler & 239.410 & 168,5 & 406,0 & 0 & 117 & 573 & 947 & 2.376 \\
Auspendler & 68.909 & 270,2 & 518,5 & 0 & 333 & 867 & 1.392 & 2.503 \\
\hline
\end{tabular}

Abbildung 2 wird - unabhängig vom jeweiligen Pendlerstatus - die Summe aller Beschäftigungszeiten zwischen dem 01.01.2000 und dem 31.12.2012 abgebildet. Abbildung 3 wertet dagegen ausschließlich solche Beschäftigungen, die dem Pendlerstatus zum 30.06.2000 entsprechen (z. B. bei einem Einpendler am 30.06.2000 nur Beschäftigungen als Einpendler zwischen dem 01.01.2000 und dem 31.12.2012). Vergleicht man die Ergebnisse aller Beschäftigungsverhältnisse in Abbildung 2 mit Beschäftigungszeiten, die ausschließlich der Wohn- und Arbeitsortkombination des 30.06.2000 entsprechen (Abbildung 3), vertauscht sich die Rangfolge der Pendlergruppen. In Bezug auf die „Statustreue" erreichen die Nichtpendler höhere Beschäftigungswerte als Einpendler. Beim Vergleich der Abbbildungen 2 und 3 wird deutlich, dass Variationen im Pendlerstatus einen positiven Beitrag zum Gesamtergebnis erzielen. Hohe Beschäftigungswerte und Dynamik im Pendlerstatus scheinen miteinander zu korrelieren.

\subsection{Arbeitslosigkeit}

Beim zweiten untersuchten Merkmal der Arbeitsmarktintegration werden Tage in registrierter Arbeitslosigkeit betrachtet. Die Unterschiede zwischen den drei Gruppen (vgl. Abbildung 4 und Tabelle 2) sind proportional größer, auch wenn die Absolutwerte deutlich kleiner ausfallen als bei der Beschäftigung. Im Durchschnitt war jeder Beschäftigte an 208 Tagen arbeitslos. Auch hier schneiden die Einpendler erneut am besten ab (168 Tage), gefolgt von den Nichtpendlern (220 Tage) und den Auspendlern (270 Tage). Insgesamt ist festzuhalten, dass Auspendler schwächer in den Arbeitsmarkt integriert sind als Einpendler und Nichtpendler.

Hinsichtlich der Betroffenheit von Arbeitslosigkeit ist der Mittelwert nicht sehr aussagekräftig, wie ein Blick auf die Verteilung in Tabelle 2 zeigt. Das Viertel mit der höchsten Betroffenheit ist etwas mehr als ein halbes Jahr arbeitslos (193 Tage), die obersten 10\% erreichen fast zwei Jahre (696 Tage) und die obersten 5\% sind sogar mehr als drei Jahre arbeitslos gewesen (1.169 Tage). Betroffene mit hohen Arbeitslosigkeitswerten üben demnach einen starken Einfluss auf den Mittelwert aus. Auch unter den Gruppen zeigen sich große Unterschiede. Das Viertel mit der höchsten Betroffenheit unter den Auspendlern ist mit etwa 11 Monaten (333 Tage) etwa dreimal so lange arbeitslos gewesen als die gleiche Gruppe unter den Einpendlern (117 Tage). Interessant ist in diesem Zusammenhang auch die Nichtbetroffenheit. Dass der Median in allen Gruppen Null beträgt (vgl. Tabelle 2), ist durch den Zuschnitt des Beschäftigtenpanels bedingt. Die Untersuchungsgruppe setzt sich aus sozialversicherungspflichtig Beschäftigten in Hamburg am 30.06.2000 zusammen. Viele dieser Beschäftigten wurden niemals arbeitslos, was die hohen Unterschiede zwischen dem Median und dem Mittelwert

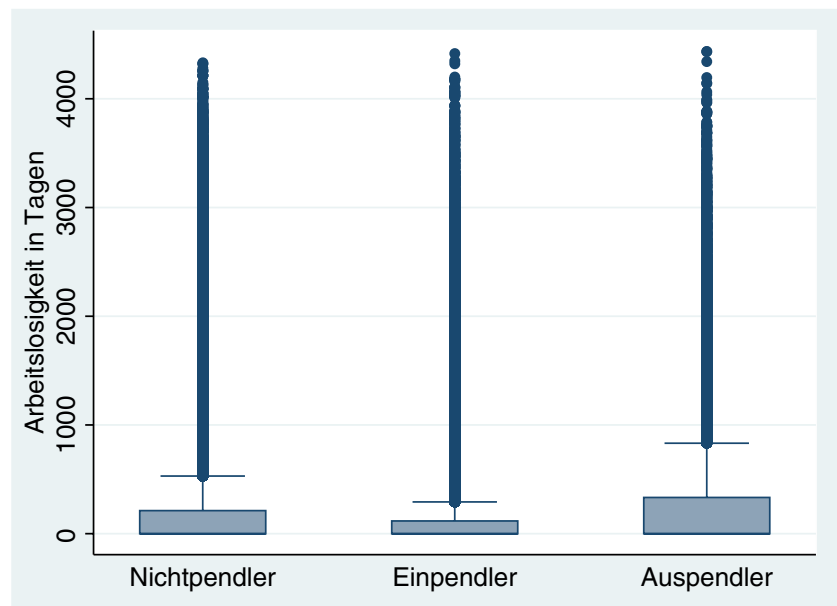

Abb. 4 Tage in Arbeitslosigkeit (Zeitraum 01.01.2000 bis 31.12.2012). Wie schon erläutert, kann der Beginn einer Arbeitslosigkeitsepisode im Einzelfall vor dem 01.01.2000 liegen. (Quelle: IEB (V11.00.00) und eigene Berechnungen) 


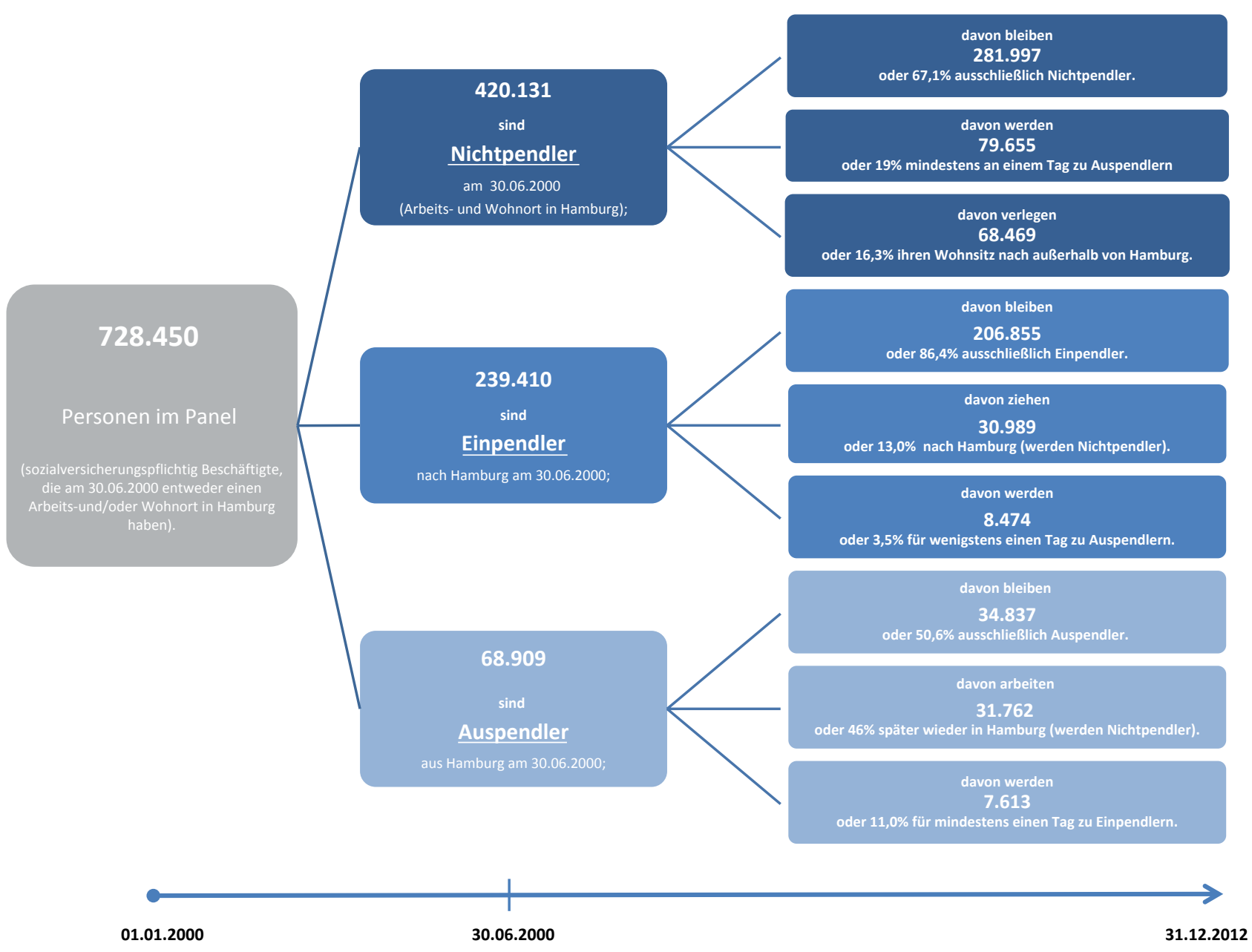

Abb. 5 Der räumliche Verbleib der Hamburger Beschäftigten. (Quelle: IEB (V11.00.00) und eigene Berechnungen)

erklärt. Im gesamten Panel ist die überwiegende Mehrheit nie arbeitslos gewesen $(62,0 \%)$, wobei Nichtpendler $(62,3 \%)$ und Einpendler (66,2\%) in etwa auf einem Niveau liegen. Unter den Auspendlern ist Arbeitslosigkeit stärker verbreitet, hier sinkt dieser Anteil der Nichtbetroffenen auf etwas mehr als die Hälfte (52,2\%). Arbeitslosigkeit ist im Ergebnis wesentlich ungleichmäßiger verteilt als Beschäftigung.

Hinsichtlich des Pendelns ist kein linearer Zusammenhang zwischen Mobilität und einer verbesserten Arbeitsmarktintegration festzustellen. In Bezug auf Beschäftigung und Arbeitslosigkeit schneiden Auspendler schlechter ab als Nichtpendler, wobei angenommen wird, dass Auspendler dabei höhere Kosten zu bewältigen haben. Bei den Einpendlern sind die Ergebnisse erwartungstreu. Deren erhöhter Aufwand ließe sich durch eine bessere Arbeitsmarktintegration erklären.

\section{Dynamik: Veränderung von Wohn- und Arbeitsorten}

\subsection{Veränderungen im Pendlerstatus}

In diesem Abschnitt geht es um den Wechsel der Wohnoder Arbeitsorte im Zeitverlauf. Dazu werden Umzüge oder Stellenwechsel, die zwischen Juni 2000 und Ende Dezember 2012 stattfanden, gesammelt ausgewertet. Im Hintergrund steht die Frage, welche Dynamik in Bezug auf Pendeln oder Nichtpendeln zu beobachten ist. Die wichtigsten Kennziffern der 728.450 Hamburger Beschäftigten stehen in Abb. 5. ${ }^{9}$ Wichtig ist: Die Summen der dritten „Spalte“ (rechts) stimmen nicht mit den Ausgangswerten vom 30.06.2000 überein (mittlere „Spalte“). Grund hierfür sind

\footnotetext{
${ }^{9}$ Für eine Zusammenstellung weiterer Kennwerte vergleiche auch Tabelle 7 im Anhang.
} 
Mehrfachzählungen. ${ }^{10}$ Für die Referenzkategorien Nichtpendler, Einpendler oder Auspendler gilt dies nicht, Doppelzählungen sind hier ausgeschlossen.

Zum Ausgangszeitpunkt, dem 30.06.2000, wohnten und arbeiteten $57 \%$ der Beschäftigten in Hamburg (420.131 Nichtpendler, vgl. im Folgenden Abbildung 5 und Tabelle 7). Etwa zwei Drittel davon behalten diesen Status bei (281.997 Personen). Etwa jeder Fünfte hat mindestens einmal den Arbeitsort verändert und außerhalb Hamburgs gearbeitet (79.655 Personen). Ein Fortzug kommt noch seltener vor, etwa jeder sechste Nichtpendler hat zumindest einmal im Umland gewohnt (68.496 Personen). Im Zusammenhang mit Verlagerungen des Wohn- oder Arbeitsortes aus Hamburg ist die Höhe der Arbeitslosigkeit auffällig. Hamburger, die eine Arbeit außerhalb der Stadt annehmen, kommen auf deutlich mehr Arbeitslosigkeitstage (421 Tage) als diejenigen, die immer in der Stadt geblieben sind (177 Kalendertage).

Die zweite Untersuchungsgruppe bilden Einpendler. Einpendler arbeiten in Hamburg und wohnen außerhalb der Stadt. Etwa jeder dritte Beschäftigte am 30.06.2000 gehört $\mathrm{zu}$ dieser Kategorie (mittlere Spalte in Abbildung 5). Ein Wechsel des Wohn- oder Arbeitsortes über die Stadtgrenze spielt für Einpendler eine geringe Rolle, fast neun Zehntel behalten die Kombination aus Wohnort im Umland und Arbeitsort in der Stadt durchgehend bei (absolut 206.855 von 239.410 Personen). Rund $11 \%$ der Einpendler sind später nach Hamburg gezogen. Mit 3.823 Beschäftigungstagen erreichen Einpendler, die nach Hamburg ziehen, den höchsten Beschäftigungswert aller Gruppen. Eine kleine Gruppe hat sogar die ursprüngliche Richtung des Pendelns vertauscht: Aus einem Einpendler wurde ein Auspendler. In dieser Gruppe ist jedoch die Arbeitslosigkeit hoch, mit 348 Kalendertagen beträgt sie fast ein ganzes Jahr.

In der Gruppe der Auspendler ist die Dynamik hinsichtlich der Wohn- und Arbeitsorte am größten. Auspendler sind Beschäftigte, die in Hamburg wohnen und für die Arbeit in das Umland pendeln. Am 30.06.2000 gehörten 68.909 Personen zu dieser Kategorie. Etwa die Hälfte von ihnen bleibt ausschließlich Auspendler (34.837 Personen). In dieser Gruppe sind die Werte der Arbeitsmarktintegration mit 3.338 Beschäftigungstagen und 158 Tagen in Arbeits-

\footnotetext{
${ }^{10}$ Beispiel: Ein Beschäftigter gehört $\mathrm{zu}$ der Gruppe der Nichtpendler am 30.06.2000 (420.131 Personen). Er nimmt später eine Beschäftigung außerhalb Hamburgs auf. Damit gehört er zu der Kategorie der 79.655 Hamburger, die mindestens einen Tag außerhalb der Hansestadt gearbeitet haben. Zu einem noch späteren Zeitpunkt wird auch der Wohnsitz in die Nähe des neuen Arbeitsortes verlegt. Damit zählt diese Person dann auch zur Gruppe der 68.470 Personen, die am 30.06.2000 in Hamburg gearbeitet und gelebt haben, aber in den Folgejahren mindestens einen Tag außerhalb Hamburgs gewohnt haben. Solche Doppelerfassungen ließen sich vermeiden, wenn das Pfaddiagramm weiter aufgeteilt würde, was aber auf Kosten der Übersichtlichkeit gehen würde.
}

losigkeit am niedrigsten. Jeder zweite Auspendler hat in späterer Zeit wieder eine Beschäftigung in Hamburg aufgenommen (31.762 Personen). Bei dieser Gruppe ist ein hoher Arbeitslosigkeitswert zu beobachten (Mittelwert 391 Tage). Etwa jeder zehnte Auspendler (7.613 Personen) hat Wohn- und Arbeitsort über die Stadtgrenze getauscht und wurde zum Einpendler.

Ein wesentliches Ergebnis dieser Verlaufsanalyse ist die Konstanz hinsichtlich der Kombination aus Wohnund Arbeitsort. Rund $72 \%$ aller Beschäftigten verbleiben in ihrer Pendlerkategorie: Nichtpendler bleiben Nichtpendler, Einpendler bleiben Einpendler, Auspendler bleiben Auspendler. Die größte Dynamik ist unter Auspendler zu beobachten, nur etwas mehr als jeder Zweite bleibt seinem ursprünglichen Status treu. Verlagerungen des Wohn- oder Arbeitsortes über die Stadtgrenze sind in der Summe - mit höheren Arbeitslosigkeitswerten verbunden. In der Literatur findet sich dazu der Hinweis, dass Arbeitslose eine individuell erhöhte Mobilität aufweisen, was den beschriebenen Zusammenhang zwischen der Veränderung des Pendlerstatus und der Betroffenheit durch Arbeitslosigkeit erklären könnte (Frick 1996: 255, ähnlich Windzio 2004: 38). Im Hinblick auf Pendlerdynamik und Beschäftigungszeiten ist ein positiver Zusammenhang nur für Einpendler zu beobachten. Betrachtet man die Arbeitsmarktintegration und die Verteilung der Wohnorte (vgl. Tabelle 7), treten zwei stabile Muster räumlicher Mobilität hervor: In der Stadt zu leben und zu arbeiten scheint eine ebenso stabile Konstellation zu sein, wie im Umland zu wohnen und in der Stadt zu arbeiten.

\subsection{Wohnortwechsel und Umzugsbereitschaft}

Wie schon erwähnt, können individuelle Entscheidungen zu pendeln oder umzuziehen mit den vorliegenden Daten nicht nachvollzogen werden. Möglich ist die Analyse einzelner personenbezogener Merkmale, die positiv oder negativ mit einem Wohnortwechsel in Verbindung stehen. Diese Ergebnisse können mit den Ergebnissen anderer Studien, die individuelle Entscheidungen einbeziehen, reflektiert werden. Im Kern geht es um die Frage, warum Beschäftigte pendeln, statt den Wohnort zu wechseln. ${ }^{11}$

Für die Analyse des Beschäftigtenpanels kommt eine Probit-Schätzung (ein ökonometrisches Schätzverfahren, mit dem der Einfluss mehrerer erklärender Variablen auf eine abhängige Variable geschätzt wird) zur Anwendung. Die Verlagerung des Wohnorts steht auf der linken Seite der Gleichung als zu erklärende Variable, während auf

\footnotetext{
${ }^{11}$ Ein Beispiel für eine umfassende qualitative Untersuchung zur berufsbedingten Mobilität liefern Schneider/Limmer/Ruckdeschel 2002. Für weitere Untersuchungen mit Bezug zu individuellen Entscheidungen vgl. Frick 1996; Kalter 1997; Windzio 2004 oder Kley 2009.
} 
Tab. 3 Modell 1, Zuzug nach Hamburg, Probit-Schätzung (alle Kategorien beziehen sich auf den Status am 30.06.2000). (Quelle: IEB (V11.00.00) und eigene Berechnungen)

\begin{tabular}{|c|c|c|c|c|}
\hline \multicolumn{2}{|l|}{ Zuzug nach Hamburg } & Koeffizient & Standardfehler & Signifikanzniveau \\
\hline \multicolumn{5}{|c|}{ (Referenzgruppe: Männer zwischen 30 und 39 Jahren mit deutscher Staatsangehörigkeit, tätig als Akademiker in einem technischen Beruf) } \\
\hline \multicolumn{5}{|l|}{ Alter } \\
\hline & 20 bis 29 Jahre & 0,1169 & 0,0023 & 0,000 \\
\hline & 40 bis 49 Jahre & $-0,0543$ & 0,0014 & 0,000 \\
\hline & 50 bis 59 Jahre & $-0,1046$ & 0,0013 & 0,000 \\
\hline \multicolumn{5}{|l|}{ Geschlecht } \\
\hline & Frauen & 0,0306 & 0,0015 & 0,000 \\
\hline \multicolumn{5}{|l|}{ Nationalität } \\
\hline & Ausländer & 0,0062 & 0,0040 & 0,000 \\
\hline \multicolumn{5}{|l|}{ Berufe } \\
\hline & $\begin{array}{l}\text { Land- und Forstwirtschaft, Fischerei, } \\
\text { Rohstoffgewinnung }\end{array}$ & 0,0618 & 0,0114 & 0,000 \\
\hline & Fertigungsleistungsberufe & 0,0276 & 0,0031 & 0,000 \\
\hline & Dienstleistungsberufe & 0,0393 & 0,0022 & 0,000 \\
\hline \multicolumn{5}{|l|}{ Berufsausbildung } \\
\hline & Mit Berufsausbildung & $-0,0306$ & 0,0016 & 0,000 \\
\hline & Ohne abgeschlossene Berufsausbildung & 0,0168 & 0,0028 & 0,000 \\
\hline \multicolumn{3}{|c|}{ Wahrscheinlichkeit der Referenzgruppe } & 0,1209 & \\
\hline \multicolumn{2}{|l|}{ Fallzahl n } & & 239.410 & \\
\hline \multicolumn{2}{|l|}{$\underline{\text { Pseudo } R^{2}}$} & & 0,0780 & \\
\hline
\end{tabular}

Tab. 4 Modell 2, Fortzug aus Hamburg, Probit-Schätzung (alle Kategorien beziehen sich auf den Status am 30.06.2000). (Quelle: IEB (V11.00.00) und eigene Berechnungen)

\begin{tabular}{|c|c|c|c|c|}
\hline \multicolumn{2}{|l|}{ Fortzug aus Hamburg } & Koeffizient & Standardfehler & Signifikanzniveau \\
\hline \multicolumn{5}{|c|}{ (Referenzgruppe: Männer zwischen 30 und 39 Jahren mit deutscher Staatsangehörigkeit, tätig als Akademiker in einem technischen Beruf) } \\
\hline \multicolumn{5}{|l|}{ Alter } \\
\hline & 20 bis 29 Jahre & 0,1158 & 0,0017 & 0,000 \\
\hline & 40 bis 49 Jahre & $-0,1010$ & 0,0013 & 0,000 \\
\hline & 50 bis 59 Jahre & $-0,1665$ & 0,0012 & 0,000 \\
\hline \multicolumn{5}{|l|}{ Geschlecht } \\
\hline & Frauen & $-0,0090$ & 0,0012 & 0,000 \\
\hline \multicolumn{5}{|l|}{ Nationalität } \\
\hline & Ausländer & $-0,1017$ & 0,0016 & 0,000 \\
\hline \multicolumn{5}{|l|}{ Berufe } \\
\hline & $\begin{array}{l}\text { Land- und Forstwirtschaft, Fischerei, } \\
\text { Rohstoffgewinnung }\end{array}$ & $-0,0376$ & 0,0060 & 0,000 \\
\hline & Fertigungsleistungsberufe & $-0,0371$ & 0,0025 & 0,000 \\
\hline & Dienstleistungsberufe & $-0,0056$ & 0,0024 & 0,019 \\
\hline \multicolumn{5}{|l|}{ Berufsausbildung } \\
\hline & Mit Berufsausbildung & $-0,0135$ & 0,0013 & 0,000 \\
\hline & Ohne abgeschlossene Berufsausbildung & $-0,0442$ & 0,0018 & 0,000 \\
\hline \multicolumn{2}{|c|}{ Wahrscheinlichkeit der Referenzgruppe } & & 0,2132 & \\
\hline \multicolumn{2}{|l|}{ Fallzahl n } & & 489.040 & \\
\hline \multicolumn{2}{|l|}{ Pseudo R ${ }^{2}$} & & 0,0630 & \\
\hline
\end{tabular}

der rechten Seite die verfügbaren Variablen aus dem Beschäftigtenpanel die „Erklärungsmenge“ bilden. Im ersten Modell werden die Zuzüge nach Hamburg betrachtet (vgl. Tabelle 3), im zweiten Modell die Fortzüge (vgl. Tabelle 4). Ausgangspunkt ist jeweils der 30.06.2000. Jede Person aus dem Beschäftigtenpanel wird zu diesem Zeitpunkt genau einer der Kategorien „Wohnort in Hamburg“ oder ,Wohnort nicht in Hamburg“" zugeordnet. Anschließend wird betrachtet, in welchen Fällen ein Wohnortwechsel über die Stadtgrenze stattfand. Beobachtetes Ereignis ist ausschließlich (anders als im vorherigen Abschnitt) der erste stattfindende Wohnortwechsel. Weitere Umzüge gehen in die Berechnung nicht mit ein. Als erklärende Variablen werden das Alter, das Geschlecht, die Nationalität, der ausgeübte Beruf und das Qualifikationsniveau berücksichtigt. Der numerische Wert beschreibt die relative (sic!) 
Abweichung gegenüber der Referenzkategorie in Prozentpunkten. Die Referenzkategorie bilden Männer zwischen 30 und 39 Jahren mit deutscher Staatsangehörigkeit, einem akademischen Abschluss und einer Beschäftigung in einem technischen Beruf. Die Auswahl der Referenzkategorie folgt heuristischen Abwägungen. Die Referenzgruppe ist durch eine positive Arbeitsmarktintegration gekennzeichnet, das heißt hohe Beschäftigungszeiten bei ausbleibenden oder nur kurzen Arbeitslosigkeitsepisoden. In diesem Sinne wird die Umzugsbereitschaft mit einer gut am Arbeitsmarkt integrierten Gruppe verglichen. Ein Lesebeispiel: Im Modell 1 des Zuzuges nach Hamburg zeigen 20- bis 29-Jährige eine höhere Bereitschaft, nach Hamburg zu ziehen, als die Referenzgruppe. Der Wert von 0,12 bedeutet, dass ein(e) 20- bis 29-Jährige(r) eine 12 Prozentpunkte höhere Umzugsbereitschaft aufweist als ein(e) 30- bis 39-Jährige(r). Umgekehrt bei über 50-Jährigen; hier liegt die Wahrscheinlichkeit, nach Hamburg zu ziehen, um 10,5 Prozentpunkte niedriger. Interessiert man sich auch für die Wirkungen mehrerer Faktoren, z. B. Qualifikation und Alter, kann man die Effekte addieren. Eine Frau im Alter von 20 bis 29 Jahren mit deutscher Staatsangehörigkeit, akademischer Qualifikation und einem technischen Beruf hat bereits eine um 14,8 Prozentpunkte höhere Neigung zum Umzug nach Hamburg als ein Mann der Referenzgruppe (Unterschiedsmerkmale: Geschlecht und Alter). Im ersten Modell der Zuzüge wird das Verhalten der 239.410 Einpendler am 30.06.2000 beobachtet. Alle Effekte in Modell eins sind hochsignifikant, die Wahrscheinlichkeit des Faktors „Zufall“ liegt bei jedem Effekt unterhalb des Ein-Prozent-Niveaus.

Mit zunehmendem Alter geht die Bereitschaft, nach Hamburg zu ziehen, deutlich zurück. Gegenüber den 30- bis 39-Jährigen liegt die Wahrscheinlichkeit eines Zuzuges unter den 40- bis 49-Jährigen um 5,4 Prozentpunkte niedriger, bei den 50 bis 59-Jährigen, wie schon erwähnt, sogar um 10,5 Prozentpunkte. Umgekehrt ist es bei Jüngeren: 20- bis 29-Jährige zeigen eine 11,7 Prozentpunkte höhere Umzugsbereitschaft als die 30- bis 39-Jährigen. Frauen haben eine etwas höhere Neigung, nach Hamburg zu ziehen, als Männer (rund 3 Prozentpunkte), Ausländer erreichen hinsichtlich des Zuzuges ein ähnliches Niveau wie deutsche Staatsangehörige. Innerhalb des Berufsspektrums haben technische Berufe die niedrigste Zuzugsbereitschaft. Die (kleine) Berufsgruppe der Beschäftigten aus der Land und Forstwirtschaft/Fischerei bzw. der Rohstoffgewinnung weist eine um 6,2 Prozentpunkte höhere Bereitschaft auf, nach Hamburg zu ziehen, als die Referenzgruppe; bei Fertigungsberufen sind es 2,7 Prozentpunkte und in der großen Gruppe der Dienstleistungsberufe 3,9 Prozentpunkte mehr. Bezogen auf das Qualifikationsniveau überrascht die Gruppe der Beschäftigten ohne Berufsausbildung; hier ist die Wahrscheinlichkeit eines Zuzuges gegenüber den Akademikern um rund 1,7 Prozentpunkte höher. Beschäftigte mit Berufsausbildung sind weniger mobil als Akademiker, ihre Zuzugswahrscheinlichkeit liegt um etwa 3 Prozentpunkte niedriger.

Im zweiten Modell wird die Bereitschaft eines Fortzuges aus Hamburg untersucht. Beobachtet werden 489.040 Beschäftigte, die am 30.06.2000 einen Wohnort in Hamburg hatten (Nichtpendler und Auspendler). Auch bei den Fortzügen ist das Alter wichtig. Bei den 20- bis 29-Jährigen ist die Umzugsbereitschaft um 11,6 Prozentpunkte höher als in der Referenzkategorie der 30- bis 39-Jährigen. Mit zunehmendem Alter nimmt dann die Fortzugsbereitschaft ab, bei den 40- bis 49-Jährigen um 10,1 Prozentpunkte und bei den 50- bis 59-Jährigen sind es dann sogar 16,7 Prozentpunkte. Ausländische Beschäftigte haben eine markant geringere Bereitschaft, Hamburg zu verlassen, ihre Fortzugswahrscheinlichkeit ist um 10,2 Prozentpunkte niedriger als bei Deutschen. Zwischen Männern und Frauen sind hinsichtlich eines Fortzuges kaum Unterschiede auszumachen. Im Berufsspektrum kommen die technischen Berufe (Referenzkategorie) auf die höchste Fortzugsbereitschaft, in der Land und Forstwirtschaft/Fischerei bzw. der Rohstoffgewinnung sind es 3,8 Prozentpunkte weniger, bei den Fertigungsberufen 3,7 und bei den Dienstleistungsberufen 0,5 Prozentpunkte weniger. Akademische Beschäftigte zeichnen sich hinsichtlich eines Fortzuges durch die höchste Mobilitätswahrscheinlichkeit aus, eine Fachkraft kommt auf etwa 1,5 Prozentpunkte weniger, An- und Ungelernte sogar auf 4,4 Prozentpunkte weniger. Während im Hinblick auf das Alter die Ergebnisse für $\mathrm{Zu}$ - und Fortzüge in eine Richtung gehen, fallen die Ergebnisse hinsichtlich der Berufe und der Qualifikation spiegelbildlich aus: Hohe Zuzugswahrscheinlichkeiten korrespondieren mit niedrigen Fortzugswahrscheinlichkeiten (mit Ausnahme der Zuzüge von Beschäftigten mit Berufsausbildung).

Diese Ergebnisse fügen sich gut in vorhandene Forschungsergebnisse ein. Das Alter zeigt sich in vielen Studien als eine wichtige Größe in Bezug auf die Umzugsbereitschaft. Allgemein gilt dabei der Zusammenhang, dass mit zunehmendem Alter die Bereitschaft zur Wohnortverlagerung zurückgeht (Kalter 1994: 473 im Hinblick auf Fernwanderungen; Frick 1996: 242 ff.; Kley 2009: 146 ff.; ähnlich Windzio 2004: 39 oder Pfaff 2013: 147). Dabei ist der Zusammenhang zwischen zunehmendem Alter und abnehmender Mobilitätsbereitschaft bei den Fortzügen aus Hamburg stärker ausgeprägt als bei den Zuzügen. Allerdings kann die Frage der Wohnortverlagerung sinnvoll nur in Verbindung mit der Betrachtung bestimmter Lebensphasen diskutiert werden (Ausbildung, Familiengründung, Übergang in den Ruhestand etc.) (vgl. Kley 2009: 221 ff.). In Haushalten mit kleinen Kindern ist die Umzugsbereitschaft geringer ausgeprägt als mit Kindern in späteren Phasen (,empty nest") oder beim Übergang in den Ruhestand (vgl. Kley 
2009). Auch strukturelle Probleme können auf das Mobilitätsverhalten bestimmter Altersgruppen einwirken. Jüngere Arbeitskräfte sind mit zunehmenden Problemen beim Eintritt in das Arbeitsleben konfrontiert. Längere Suchphasen, hohe Jugendarbeitslosigkeit, befristete Beschäftigung usw. bilden Rahmenbedingungen, die räumliche Mobilität verstärken (Buchholz/Kurz 2008: 68). Diese Einflüsse konnten im Modell nicht nachgebildet werden. Beim Merkmal Geschlecht zeigt sich in dieser Untersuchung ein auffälliger Effekt. Frauen zeigen eine höhere Bereitschaft, in die Stadt zu ziehen, als diese zu verlassen. Ähnliches lässt sich für Ausländer beobachten, bei ihnen ist die Fortzugsbereitschaft wesentlich schwächer ausgeprägt als bei Deutschen. Bei der Qualifikation ist grundsätzlich ein positiver Zusammenhang zwischen dem Bildungsniveau und der Umzugsbereitschaft festzustellen; auch dieses Ergebnis wurde bereits in verschiedenen Studien beschrieben (vgl. dazu Frick 1996: 252 ff.; Kley 2009: 146 oder Pfaff 2012: 468 ff.).

Fasst man die Beobachtungen zusammen, wird deutlich, dass die ausgewiesenen Effekte nicht isoliert betrachtet werden dürfen. Es ist auch davon auszugehen, dass andere wichtige Einflüsse im Modell nicht erfasst sind. Strukturelle Faktoren wie die Wohnsituation oder die individuelle Arbeitsmarktposition spielen für die Umzugsentscheidung ebenso eine Rolle wie die hier erfassten Eigenschaften.

\section{Fazit}

Zwei Befunde aus der Untersuchung sollen abschließend unterstrichen werden. Räumliche Mobilität hat einen positiven Bezug zur Arbeitsmarktintegration, aber der Zusammenhang ist nicht in jedem Fall eindeutig. Dass Nichtpendler im Durchschnitt länger beschäftigt und kürzer arbeitslos sind als Auspendler, also im Vergleich besser abschneiden, war nicht zu erwarten. Auch wenn eine Ergänzung der Untersuchung um die Entfernungsangabe zwischen Wohn- und Arbeitsort aussteht, ist davon auszugehen, dass Auspendler im Durchschnitt weitere Wege zurücklegen. Insofern ist der unterdurchschnittliche Erfolg der Auspendler überraschend. Die Ergebnisse zeigen auch, dass diejenigen, die ihren Pendlerstatus verändern, das heißt zwischen Pendlerformen wechseln, einerseits höhere Beschäftigungszeiten erreichen, aber andererseits auch längere Arbeitslosigkeitsepisoden aufweisen. Folglich kann die Annahme eines eindeutigen Zusammenhanges zwischen räumlicher Mobilität und der Arbeitsmarktintegration durch die Befunde nicht bestätigt werden.
Das zweite Resultat betrifft die Umzugsbereitschaft. Hier steht die große Konstanz bei der Wahl der Wohnund Arbeitsorte im Vordergrund. Im Beobachtungszeitraum bleiben die meisten Pendler ihrem Status treu, die überwiegende Mehrzahl der Einpendler bleibt Einpendler, Nichtpendler bleiben Nichtpendler und - am geringsten ausgeprägt - Auspendler bleiben Auspendler. Wie schon erwähnt ist die Arbeitsmarktintegration bei Auspendlern schwächer ausgeprägt als bei den beiden anderen Gruppen. Leben und Arbeiten in Hamburg scheint eine ebenso attraktive Konstellation zu sein (Nichtpendler) wie Wohnen im Umland und Arbeiten in der Stadt (Einpendler). Bezieht man die Befunde zur Umzugsbereitschaft mit ein (ProbitModell), offenbaren sich weitere Ergebnisse, die sich in die bisherigen Resultate der Mobilitätsforschung gut einfügen. Auch bei den Hamburger Beschäftigten ist als wichtiger Einfluss hinsichtlich der Umzugsbereitschaft das Alter zu beobachten, Ähnliches gilt in Bezug auf das Geschlecht und die Nationalität. Interessant sind die Unterschiede in der Richtung des Umzuges. Unter Frauen ist die Zuzugsbereitschaft nach Hamburg stärker ausgeprägt als bei Männern; Ausländer zeigen eine deutlich geringere Bereitschaft, Hamburg zu verlassen, als Deutsche. Hinsichtlich der Effekte des Berufes und der Qualifikation wäre eine weitere Vertiefung sinnvoll. Hier sind weitere Differenzierungen zu erwarten. Bei genauer Betrachtung zerfällt berufsbedingtes Pendeln in ein Mosaik aus Einzelformen, das durch individuelle, strukturelle und lebenszyklusbezogene Faktoren beeinflusst wird.

\section{Einhaltung ethischer Richtlinien}

Interessenkonflikt Es besteht kein Interessenkonflikt. 


\section{Anhang}

Tab. 5 Beschäftigte im Panel nach Alter und Geschlecht (Stichtag: 30.06.2000). (Quelle: IEB (V11.00.00) und eigene Berechnungen)

\begin{tabular}{lllllllll}
\hline & $\begin{array}{l}\text { Alter (Mittelwert) } \\
\text { insgesamt }\end{array}$ & $N$ & \multicolumn{2}{c}{$\begin{array}{l}\text { Alter (Mittel- } \\
\text { wert) Männer }\end{array}$} & in \% & \multicolumn{2}{c}{$\begin{array}{c}\text { Alter (Mittel- } \\
\text { wert) Frauen }\end{array}$} & in \% \\
\hline Nichtpendler & $\mathbf{3 8 , 8}$ & $\mathbf{4 2 0 . 1 3 1}$ & $\mathbf{3 9 , 0}$ & $\mathbf{2 2 1 . 7 9 1}$ & $(52,8 \%)$ & $\mathbf{3 8 , 6}$ & $\mathbf{1 9 8 . 3 4 0}$ & $(47,2 \%)$ \\
Einpendler & $\mathbf{3 9 , 7}$ & $\mathbf{2 3 9 . 4 1 0}$ & $\mathbf{4 0 , 6}$ & $\mathbf{1 6 0 . 2 6 3}$ & $(66,9 \%)$ & $\mathbf{3 8 , 0}$ & $\mathbf{7 9 . 1 4 7}$ & $(33,1 \%)$ \\
Auspendler & $\mathbf{3 7 , 6}$ & $\mathbf{6 8 . 9 0 9}$ & $\mathbf{3 8 , 3}$ & $\mathbf{4 5 . 4 2 2}$ & $(65,9 \%)$ & $\mathbf{3 6 , 3}$ & $\mathbf{2 3 . 4 8 7}$ & $(34,1 \%)$ \\
Panel (insgesamt) & $\mathbf{3 9 , 0}$ & $\mathbf{7 2 8 . 4 5 0}$ & $\mathbf{3 9 , 6}$ & $\mathbf{4 2 7 . 4 7 6}$ & $(58,7 \%)$ & $\mathbf{3 8 , 3}$ & $\mathbf{3 0 0 . 9 7 4}$ & $(41,3 \%)$ \\
\hline
\end{tabular}

Tab. 6 Beschäftigte nach Qualifikation und Kategorie (maximales Qualifikationsniveau im Panel-Zeitraum; Stichtag: 30.06 .2000 ). (Quelle: IEB (V11.00.00) und eigene Berechnungen)

\begin{tabular}{|c|c|c|c|c|c|c|}
\hline & & Unbekannt & Ohne Berufsausbildung & Mit Berufsausbildung & FH/Uni & Total \\
\hline Nichtpendler & Insgesamt & $3,5 \%$ & $10,6 \%$ & $66,2 \%$ & $19,8 \%$ & $100,0 \%$ \\
\hline Nichtpendler & Männer & $3,5 \%$ & $10,5 \%$ & $64,0 \%$ & $22,1 \%$ & $100,0 \%$ \\
\hline Nichtpendler & Frauen & $3,4 \%$ & $10,6 \%$ & $68,6 \%$ & $17,3 \%$ & $100,0 \%$ \\
\hline Einpendler & Insgesamt & $2,6 \%$ & $4,5 \%$ & $70,8 \%$ & $22,2 \%$ & $100,0 \%$ \\
\hline Einpendler & Männer & $2,6 \%$ & $4,1 \%$ & $68,3 \%$ & $25,1 \%$ & $100,0 \%$ \\
\hline Einpendler & Frauen & $2,5 \%$ & $5,4 \%$ & $75,8 \%$ & $16,3 \%$ & $100,0 \%$ \\
\hline Auspendler & Insgesamt & $3,4 \%$ & $8,9 \%$ & $60,6 \%$ & $27,1 \%$ & $100,0 \%$ \\
\hline Auspendler & Männer & $3,6 \%$ & $8,8 \%$ & $59,9 \%$ & $27,7 \%$ & $100,0 \%$ \\
\hline Auspendler & Frauen & $3,0 \%$ & $9,1 \%$ & $62,0 \%$ & $25,9 \%$ & $100,0 \%$ \\
\hline Panel & Insgesamt & $3,2 \%$ & $8,4 \%$ & $67,2 \%$ & $21,3 \%$ & $100,0 \%$ \\
\hline Panel & Männer & $3,2 \%$ & $7,9 \%$ & $65,1 \%$ & $23,8 \%$ & $100,0 \%$ \\
\hline Panel & Frauen & $3,1 \%$ & $9,1 \%$ & $70,0 \%$ & $17,7 \%$ & $100,0 \%$ \\
\hline
\end{tabular}

Hinweis: Ein Problem der Beschäftigtenstatistik ist das Qualifikationsniveau. Je nach Auswahl und Zuschnitt (Beruf, Alter, Wirtschaftszweig etc.) ist bei bis zu einem Drittel der Beschäftigten die ausgewiesene Qualifikation nicht valide. Für den Stichtag 30.06.2000 trifft das etwa auf jeden Fünften (18,3\%) zu. Vorteil des Untersuchungspanels ist die Betrachtung von Zeiträumen. Mit dem „Maximierungsverfahren“ wird das höchste gültige Qualifizierungsniveau im Untersuchungszeitraum ermittelt. Dabei kann der Anteil des unbekannten Qualifikationsniveaus auf $3,2 \%$ begrenzt werden. Trotzdem sei auf mögliche Probleme dieser Vorgehensweise hingewiesen. Im Fall einer späteren Höherqualifizierung oder dem Nachholen eines Ausbildungsabschlusses besteht das Risiko, diese Information rückwirkend auf die gesamte Erwerbsbiographie zu übertragen. Im Extremfall kann ein Beschäftigter, der erst im Jahr 2012 einen Berufsabschluss nachholt, so behandelt werden, als ob er bereits seit dem Jahr 2000 über eine berufliche Qualifikation verfügt. Umgekehrt gilt dies auch für das Minimierungsprinzip, bei dem ein Beschäftigter, der nur am 30.06.2000 keine Qualifikationsangabe hat, durchgehend als Ungelernter betrachtet würde. Bei einer manuellen Inspektion fraglicher Fälle zeigte sich, dass die Probleme beim Maximierungsverfahren als nicht gravierend einzuschätzen sind. In den meisten Fällen liegen valide und widerspruchsfreie Informationen zu späteren Zeitpunkten vor, der potenzielle Informationsgewinn dürfte damit das Risiko einer Überschätzung übersteigen 


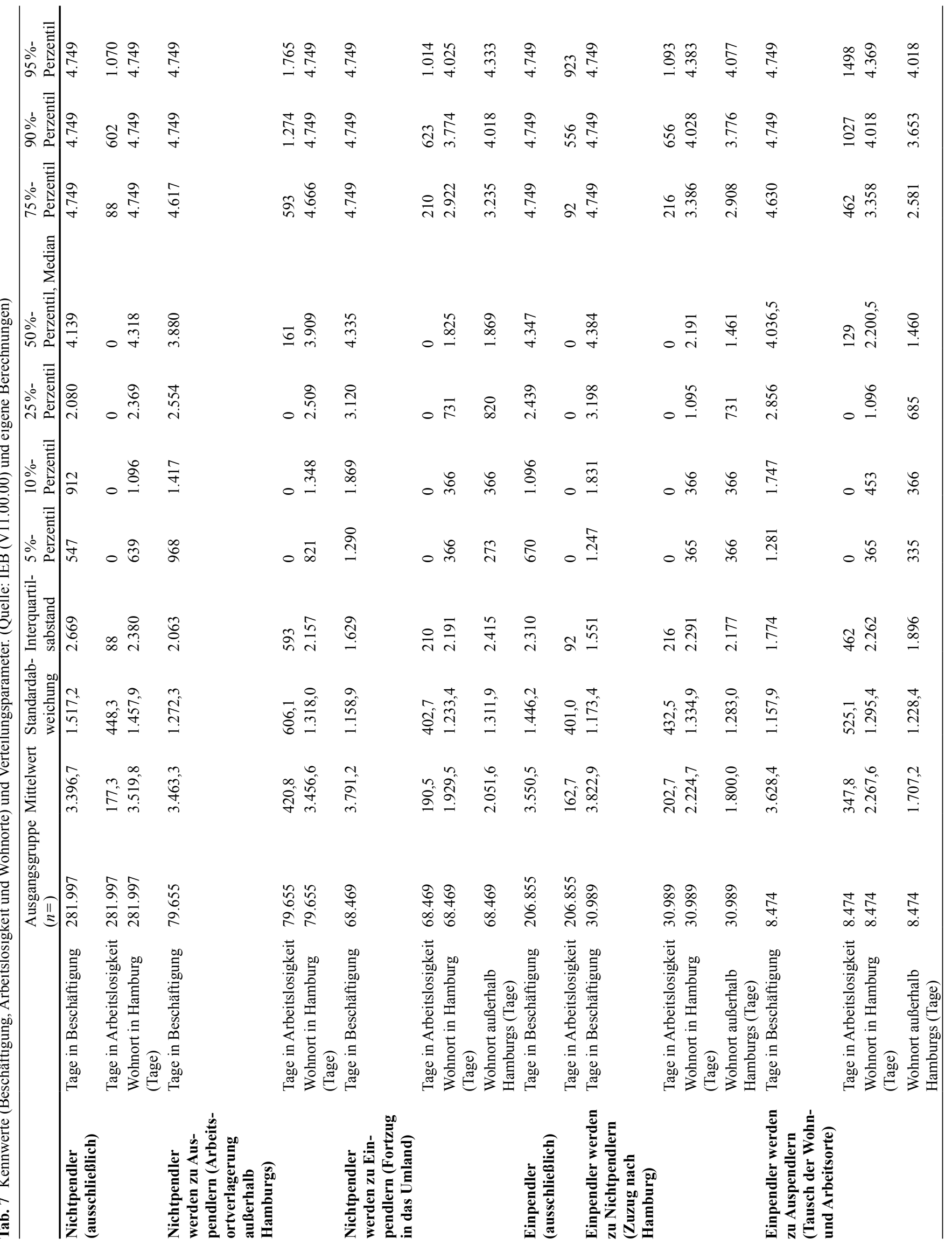




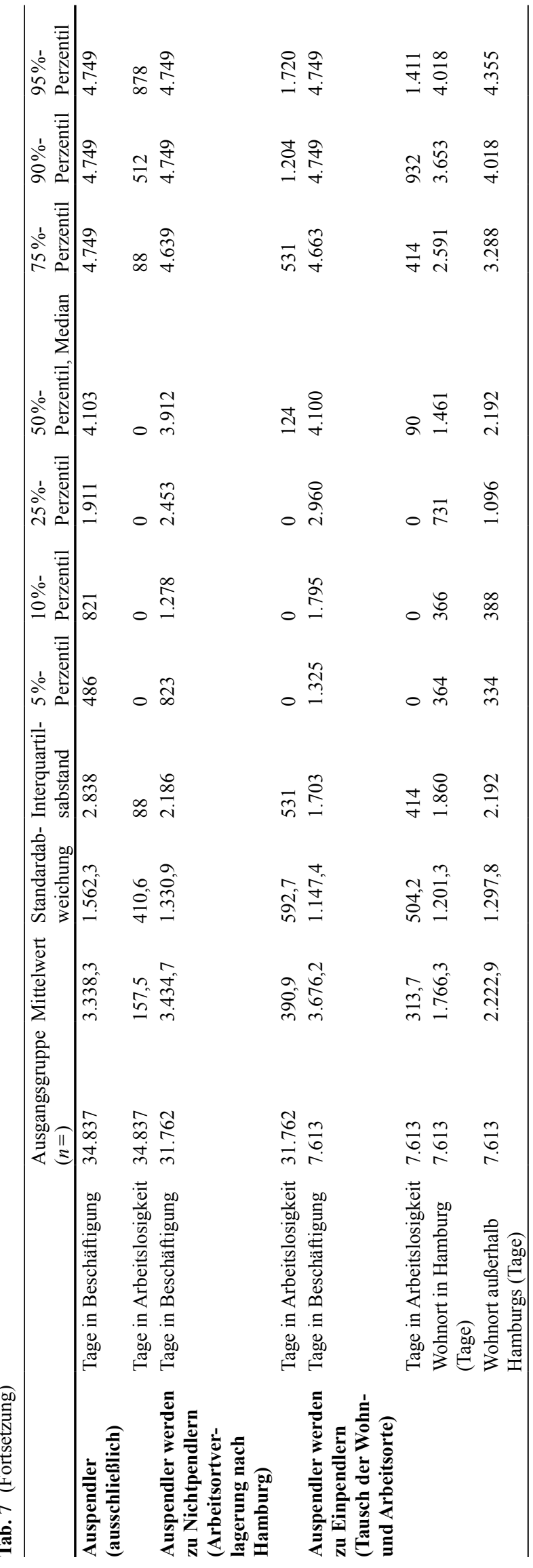

\section{Literatur}

Abraham, M.; Nisic, N. (2007): Regionale Bindung, räumliche Mobilität und Arbeitsmarkt - Analysen für die Schweiz und Deutschland. Schweizerische Zeitschrift für Soziologie 33, 1, 69-87.

Abraham, M.; Schönholzer, T. (2009): Pendeln oder Umziehen? Entscheidungen über unterschiedliche Mobilitätsformen in Paarhaushalten. In: Kriwy, P.; Gross, C. (Hrsg.): Klein aber fein! Quantitative empirische Sozialforschung mit kleinen Fallzahlen. Wiesbaden, 247-268.

Beck, U.; Bonß, W. (Hrsg.) (2001): Die Modernisierung der Moderne. Frankfurt am Main.

Buch, T.; Hamann, S.; Niebuhr, A. (2010): Qualifikationsspezifische Wanderungsbilanzen deutscher Metropolen: Hamburg im Städtevergleich. Nürnberg. = IAB Regional. Berichte und Analysen aus dem Regionalen Forschungsnetz (IAB Nord, 02/2010).

Buchholz, S.; Kurz, K. (2008): A new mobility regime in Germany? Young people's labor market entry and phase of establishment since the mid-1980s. In: Blossfeld, H.-P.; Buchholz, S.; Bukodi, E.; Kurz, K. (Hrsg): Young Workers, Globalization and the Labor Market. Comparing Early Working Life in Eleven Countries. Cheltenham, Northampton, 51-75.

Bundesagentur für Arbeit (2014): Arbeitsmarkt 2013. Arbeitsmarktanalyse für Deutschland, West- und Ostdeutschland. = Amtliche Nachrichten der Bundesagentur für Arbeit 61 (Sondernummer 2).

Bundesagentur für Arbeit (2016): Arbeitsmarkt in Zahlen Beschäftigungsstatistik. Länderreport Hamburg (Stichtag 30.06.2015). Hannover.

Einig, K.; Pütz, T. (2007): Regionale Dynamik der Pendlergesellschaft. Entwicklung von Verflechtungsmustern und Pendeldistanzen. In: Informationen zur Raumentwicklung 2-3/2007, 73-91.

Feldhaus, M.; Schlegel, M. (2013): Berufsbezogene zirkuläre Mobilität und Partnerschaftszufriedenheit. In: Comparative Population Studies - Zeitschrift für Bevölkerungswissenschaft 38, 2, 315-340.

Frick, J. (1996): Lebenslagen im Wandel: Determinanten kleinräumlicher Mobilität in Westdeutschland. Frankfurt am Main, New York. $=$ Sozio-ökonomische Daten und Analysen für die Bundesrepublik Deutschland, 9 (Dissertation).

Fuchs, J.; Walwei, U.; Weber, B. (2005): Die „Stille Reserve“ gehört ins Bild vom Arbeitsmarkt. = IAB-Kurzbericht 21/2005.

Granato, N.; Haas, A.; Hamann, S.; Niebuhr, A. (2009): Arbeitskräftemobilität in Deutschland. Qualifikationsspezifische Befunde regionaler Wanderungs- und Pendlerströme. In: Raumforschung und Raumordnung 67, 1, 21-33.

Guth, D.; Siedentop, S.; Holz-Rau, C. (2012): Erzwungenes oder exzessives Pendeln? Zum Einfluss der Siedlungsstruktur auf den Berufspendelverkehr. In: Raumforschung und Raumordnung 70, $6,485-499$

Haas, A.; Hamann, S. (2008): Pendeln - ein zunehmender Trend, vor allem bei Hochqualifizierten. Ost-West-Vergleich. = IAB-Kurzbericht $06 / 2008$.

Herrmann, H. (2009): Zentripetale Berufspendlerströme in SchleswigHolstein/Hamburg: Ergebnisse von Gravitationsregressionen sowie Anmerkungen zur Schätzung grenzüberschreitender Pendlermobilität im deutsch-dänischen Grenzraum. Vortrag, gehalten am 27.03.2009 in Dortmund. http://www.vpl.tudortmund.de/pdf/pendlerwokshop/Praesentation_Herrmann.pdf (26.01.2016).

Herrmann, H.; Schulz, A.-C. (2005): Räumliches Muster der Berufspendlerverflechtung im Raum Schleswig-Holstein/Hamburg. Kreis- und gemeindeüberschreitende Pendlerströme im Zeitraum 1993 bis 2003. Kiel. = Beiträge aus dem Institut für Regionalforschung der Universität Kiel, 40. 
Holtermann, L.; Otto, A.; Schulze, S. (2013): Haspa Hamburg-Studie. L(i)ebenswertes Hamburg. Berufspendler in der Metropolregion, herausgegeben von der Hamburger Sparkasse AG, erstellt vom Hamburgischen WeltWirtschaftsInstitut (HWWI) unter Mitarbeit von A. Lagemann und S. Tonn. Hamburg.

Kalter, F. (1994): Pendeln statt Migration? Die Wahl und Stabilität von Wohnort-Arbeitsort-Kombinationen. In: Zeitschrift für Soziologie $23,6,460-476$.

Kalter, F. (1997): Wohnortwechsel in Deutschland. Ein Beitrag zur Migrationstheorie und zur empirischen Anwendung von RationalChoice-Modellen. Wiesbaden.

Kley, S. (2009): Migration im Lebensverlauf. Der Einfluss von Lebensbedingungen und Lebenslaufereignissen auf den Wohnortwechsel. Wiesbaden.

Kotte, V. (2008): Ausbildungsmobilität in der Freien und Hansestadt Hamburg. Nürnberg. $=$ IAB regional. Berichte und Analysen $(\mathrm{IAB}$ Nord, 02/2008)

Kotte, V. (2011): Junge Berufseinsteiger aus Mecklenburg-Vorpommern. Bleiben, Gehen und Rückkehr. In: Zeitschrift für Berufs- und Wirtschaftspädagogik 107, 1, 93-108.

Niebuhr, A.; Kotte, V. (2009): Regionale Arbeitskräftemobilität: Bundesweite Trends und aktuelle Befunde für die Hansestadt Hamburg. Nürnberg. = IAB Regional. Berichte und Analysen aus dem Regionalen Forschungsnetz (IAB Nord, 01/2009).

Pfaff, S. (2012): Pendeln oder umziehen? Mobilitätsentscheidungen in Deutschland zwischen 2000 und 2009. In: Zeitschrift für Soziologie 41, 6, 458-476.
Pfaff, S. (2013): Pendeln oder umziehen? Ursachen und Folgen berufsbedingter räumlicher Mobilität in Deutschland. Karlsruhe.

Pfaff, S. (2014): Pendelentfernung, Lebenszufriedenheit und Entlohnung. Eine Längsschnittuntersuchung mit den Daten des SOEP von 1998 bis 2009. In: Zeitschrift für Soziologie 43, 2, 113-130.

Ruppenthal, S.; Lück, D. (2009): Jeder fünfte Erwerbstätige ist aus beruflichen Gründen mobil: Berufsbedingte räumliche Mobilität im Vergleich. In: Informationsdienst Soziale Indikatoren 42/2009, $1-5$.

Schneider, N. F.; Limmer, R.; Ruckdeschel, K. (2002): Berufsmobilität und Lebensform. Sind berufliche Mobilitätserfordernisse in Zeiten der Globalisierung noch mit der Familie vereinbar? Stuttgart. = Schriftenreihe des Bundesministeriums für Familie, Senioren, Frauen und Jugend, 208.

Schulz, A.-C.; Bröcker, J. (2007): Die Erreichbarkeit der Arbeitsmärkte für Berufspendler aus den Gemeinden SchleswigHolsteins. Nürnberg. $=\mathrm{IAB}$ regional. Berichte und Analysen $(\mathrm{IAB}$ Nord, 01/2007).

Van der Loo, H.; van Reijen, W. (1992): Modernisierung. Projekt und Paradox. München.

Windzio, M. (2004): Zwischen Nord- und Süddeutschland: Die Überwindung räumlicher Distanzen bei der Arbeitsmarktmobilität. In: Zeitschrift für Arbeitsmarktforschung 37, 1, 29-44. 